\title{
Evaluation of hydrodynamic conditions for journal bearings under static load
}

\section{Douglas J. Ramos (IC), Gregory B. Daniel (PQ)}

\section{Abstract}

This project presents the results obtained from the numerical solution of Reynolds equation in static condition. A computational code was developed in FORTRAN in order to obtain the pressure distribution and, consequently, the hydrodynamic forces in the journal bearing. Finally, the locus of the shaft inside the bearing can be obtained from the balance of force for each rotational speed.

Key words: Hydrodynamic Lubrication, Journal Bearing, Numerical Solution.

\section{Introduction}

Hydrodynamic lubrication is present in many machines and joints. Thus, the evaluation of the hydrodynamic forces is important to describe the hydrodynamic conditions in these machines. In this work, the Reynolds equation is numerically solved for static conditions in order to obtain the pressure distribution in the journal bearing. Then, the hydrodynamic forces are calculated and the balance of forces can be performed in order to determine the shaft position inside the bearing. Therefore, the minimum film thickness and the hydrodynamic conditions can be evaluated for different operational conditions in the bearing.

\section{Results and Discussion}

The theoretical base of the hydrodynamic lubrication was developed by Reynolds in 1886.

The classical Reynolds equation for static case is presented as:

$$
\frac{\partial}{\partial x}\left(h^{3} \frac{\partial p}{\partial x}\right)+\frac{\partial}{\partial z}\left(h^{3} \frac{\partial p}{\partial z}\right)=6 \mu U \frac{d h}{d x}
$$

This equation is a partial differential equation and does not present a complete analytical solution. For this reason, the finite volume method is used to solve the Reynolds equation in order to obtain the pressure distribution in the bearing. Fig. (1) shows the pressure distribution obtained in the bearing.

Using the pressure in the mesh points of the program, the hydrodynamic forces were calculated and used to perform a force balancing, searching the equilibrium point of the shaft. Fig. (2) shows the locus of the shaft inside the bearing, considering the ratio between length and diameter (L/D) of 0.5 .

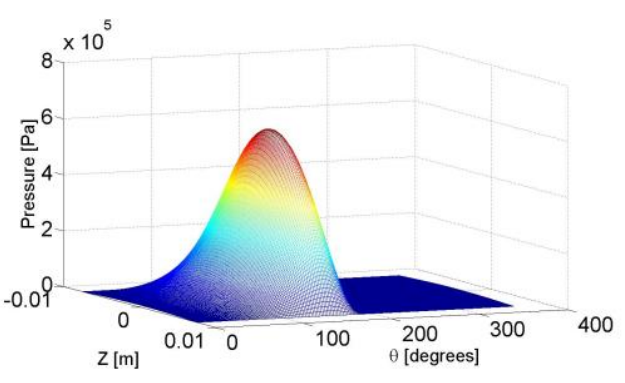

Figure 1. Pressure distribution in bearing with $L / D$ ratio of 0.5 , load of $100 \mathrm{~N}$ and rotation of $3000 \mathrm{rpm}$

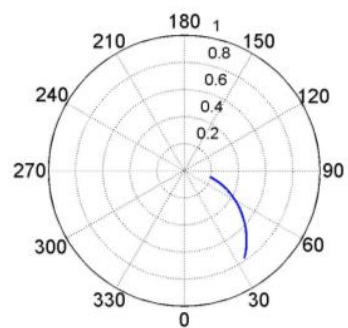

Figure 2. Locus of the shaft with $L / D$ ratio of 0.5 obtained from $1000 \mathrm{rpm}$ to $20000 \mathrm{rpm}$.

\section{Conclusions}

From results obtained in this work, an evaluation of the hydrodynamic forces and the shaft position inside the bearing were accomplished. Therefore, it is possible to conclude that the computational code developed in this work can be used efficiently to predict the hydrodynamic conditions in journal bearing.

\section{Acknowledgement}

The authors would like to thank SAE, Unicamp and ThyssenKrupp for research industry scholarship programme.

\footnotetext{
${ }^{1}$ Reynolds, O., "On the Theory of Lubrication and its Application to Mr. Beauchamp Tower's Experiments, including an Experimental Determination of the Viscosity of Olive Oil", Philos.Transactions of Royal Society of London, Series A, Vol. 177, Part 1, 1886.

2 Norton, R. L., "Machine Design: An Integrated Approach" (in Portuguese), $2^{\text {a }}$ edição, Bookman, Porto Alegre, Brazil, 2004.
} 